Mixed-Model

\section{Multiple-Hypothesis Tracking of Targets in Clutter}

PETER S. MAYBECK, Fellow, IEEE

\author{
MATTHEW C. KOZAK
}

BRIAN D. SMITH

Air Force Institute of Technology

Tracking targets in clutter, with the inherent data association problem, naturally leads to a Gaussian mixture representation of the probability density function (pdf) of the target state vector, conditioned on the measurements observed. Online trackers require reduction of the number of components in the mixture on each processing cycle, and the integral square error (ISE) based mixture reduction algorithm (MRA) significantly outperforms known alternative algorithms. Moreover, to handle target maneuver onset and changing trajectory characteristics, one can use multiple model adaptive estimation in the form of either multiple model adaptive estimation (MMAE) or interacting multiple model (IMM) algorithms. For maneuvering targets in clutter, one can replace each Kalman filter within a conventional MMAE or IMM with an ISE-based MRA, or better yet, replace each Kalman filter within an ISE-based algorithm with an MMAE or IMM, to yield superior tracking of aggressive maneuvers in deep clutter. Such an ISE-based algorithm of MMAEs is seen to have performance attributes significantly superior to that of a current state-of-the-art tracker.

Manuscript received June 28, 2006; revised September 28, 2006 and June 27, 2007; released for publication August 23, 2007.

IEEE Log No. T-AES/44/4/930720.

Refereeing of this contribution was handled by W. Koch.

The views expressed in this article are those of the authors and do not reflect the official policy or position of the United States Air Force, the Department of Defense, or the U.S. Government.

Authors' address: Dept. of Electrical Engineering, Air Force Institute of Technology, 2950 Hobson Way, Bldg. 640, Wright-Patterson AFB, OH 45433-7765, E-mail: (Peter.Maybeck@afit.edu).

U.S. Government work not protected by U.S. copyright.

0018-9251/08/\$25.00 (c) 2008 IEEE

\section{INTRODUCTION}

When tracking a target in clutter, one must deal with the data association problem [2] of whether a particular measurement is target originated or clutter originated. This leads naturally to a Gaussian mixture (probability-weighted sum of Gaussians) representation of the probability density function (pdf) of the target state vector (composed of positions, velocities, accelerations, etc.), conditioned on the measurements that have been observed, with each component of the mixture being associated with a particular association history hypothesis [1]. Due to the exponential increase in such hypotheses over time, this requires reduction of the number of components in the mixture on each processing cycle. Techniques such as joint probability data association (JPDA) [3:310-319] and global nearest neighbor (GNN) [5:338-342] perform a vast simplification, reducing the entire Gaussian mixture to a single Gaussian component. State-of-the-art multiple hypothesis tracker (MHT) algorithms [4:283-300, 6, 7] and Salmond's joining and clustering filters [18-21] maintain the mean, covariance and probability weight corresponding to each association history hypothesis, yet they rely on ad hoc merging and pruning rules to control the growth of hypotheses. This can cause severe degradation in performance. A more structured approach reduces the number of components in the Gaussian mixture on each processing cycle by minimizing a distance or cost function that measures the difference between the Gaussian mixture pdf surfaces before and after that reduction in number of components. This concept has been significantly enhanced by using an integral square error (ISE) distance metric that, unlike any previously proposed metrics, requires neither numerical integration nor approximation for evaluation [27, 28]. The performance of such an algorithm for tracking a single target in heavy clutter has been thoroughly investigated $[27,28]$ and found to surpass that of other algorithms by a significant margin.

An extension of the ISE-based MHT algorithm (for handling clutter) is made for targets exhibiting substantially different trajectory characteristics (such as due to the onset of jinking maneuvers) during the tracking scenario. One potentially useful tracker architecture would replace each Kalman filter within a conventional multiple model adaptive estimator (MMAE) or interacting multiple model (IMM) algorithm with an ISE-based algorithm that assumes the adequacy of the same particular dynamics model and discretized parameter choice ("mode") $[13,24]$. Analyses have indicated the extreme importance of proper gating for maximum performance benefit, and resolving that issue well has resulted in a superior architecture of replacing each Kalman filter within the ISE-based algorithm (each assuming a particular 
association history hypothesis is true) with an MMAE or IMM $[8,9]$. This yields the ability to track an aggressively maneuvering target in deep clutter with exceptionally small $\mathrm{rms}$ errors and probability of track loss.

An alternative state-of-the-art approach to this problem is JPDA combined with IMM [5, 14]. JPDA can be likened in concept and performance to using Gaussian mixture reduction but reducing the original mixture to only one Gaussian component (versus, for example, 15 as in this research), giving up significant performance potential by such a crude approximation. For instance, in a very heavy clutter environment (two orders of magnitude denser than used herein), it was outperformed by a tracker based on an ISE Gaussian mixture reduction to 15 components by more than an order-of-magnitude increase in average track life [27], the most appropriate figure of merit in such an environment [18-21, 27, 28]. Of available Gaussian mixture reduction techniques, the ISE algorithm has been shown to be superior, as discussed further at the end of Section II. With regard to IMM versus MMAE, despite claims in the open literature [e.g., 3, 5, 7, 14] of the superiority of IMM and the superseding of MMAE by IMM, this research provides a strictly fair head-to-head comparison of the two algorithms and demonstrates that, in this physically meaningful application, the MMAE consistently outperforms the IMM by a substantial margin. This is shown fully in Section V.

\section{GAUSSIAN MIXTURES AND DATA ASSOCIATION}

The tracking system is provided with a set of detections, each of which indicates the possible presence of a target. However, the system does not know which measurement belongs to a target or which measurements are actually false alarms (the result of radar clutter); the target/measurement association history over time is unknown. If the association history were known, a Kalman filter could produce the optimum estimation of the target (for linear dynamics and measurement models).

A Gaussian mixture, consisting of a probabilityweighted sum of Gaussian pdfs, each with mean and covariance as computed by a Kalman filter based on an assumed association history, is the natural form of pdf of the target state in this problem [27, 28]. To see this, assume that at each time instant $k$, we receive a set of measurements $\mathbf{Z}_{k}$, which may or may not contain a target-originated measurement, along with zero or more clutter measurements (false alarms). Denoting the measurement history up to time instant $(k-1)$ as $\mathbf{Z}^{k-1}=\left\{\mathbf{Z}_{1}, \ldots, \mathbf{Z}_{k-1}\right\}$, the a priori pdf (i.e., prior to introduction of the set of measurements at time instant $k$ ) of the target state $\mathbf{x}$ at time instant $k$ can be written using a total probability expansion over the $N_{h}(k-1)$ association history hypotheses
$\left\{\boldsymbol{\Psi}_{u}(k-1) \mid u=1,2, \ldots, N_{h}(k-1)\right\}$ from the previous processing cycle as

$$
\begin{aligned}
& f\left\{\mathbf{x}(k) \mid \mathbf{Z}^{k-1}\right\} \\
& \quad=\sum_{u=1}^{N_{h}(k-1)} f\left\{\mathbf{x}(k) \mid \mathbf{Z}^{k-1}, \Psi_{u}(k-1)\right\} P\left\{\Psi_{u}(k-1) \mid \mathbf{Z}^{k-1}\right\}
\end{aligned}
$$

where $P\left\{\boldsymbol{\Psi}_{u}(k-1) \mid \mathbf{Z}^{k-1}\right\}$ is the probability of the $u$ th association history hypothesis (for $u=1,2, \ldots$, $N_{h}(k-1)$ ), and $f\left\{\mathbf{x}(k) \mid \mathbf{Z}^{k-1}, \Psi_{u}(k-1)\right\}$ is the target state pdf conditioned on the $u$ th association history hypothesis. If the pdf at the previous time index $(k-1)$ conditioned on a specific association history hypothesis $f\left\{\mathbf{x}(k-1) \mid \mathbf{Z}^{k-1}, \Psi_{u}(k-1)\right\}$ is Gaussian and a linear dynamics model sufficiently describes the real-world target, then the standard Kalman filter propagation equation can be used to calculate the corresponding $f\left\{\mathbf{x}(k) \mid \mathbf{Z}^{k-1}, \Psi_{u}(k-1)\right\}$, which is also Gaussian. (Note that, with nonlinear models, an extended Kalman filter could be used instead, and the density can be approximated as Gaussian. In this research, it is assumed that linear constant-acceleration flight dynamics in a Cartesian plane is an adequate model, and that direct noise-corrupted measurements of those Cartesian coordinates are available, versus range and angles, so that nonlinear geometric relations are not required.)

In order to introduce the set of $N_{m}(k)$ measurements at time instant $k$ (i.e., $\mathbf{Z}_{k}$ ), a disjoint partitioning of the probability space $\left\{\psi_{0}(k), \ldots, \psi_{N m}(k)\right\}$ is introduced, in which $\psi_{0}(k)$ denotes the event proposing that the target was not detected at time instant $k$ (and therefore that all $N_{m}(k)$ measurements in $\mathbf{Z}_{k}$ are the result of clutter), and $\psi_{i}(k)$ for $i=1,2, \ldots, N_{m}(k)$ denotes the event proposing that measurement $i$ originated from the target (and the other measurements originated from clutter). The pdf of the target state at time instant $k$ conditioned on the new measurement history can be evaluated through the double-expansion over previous association history hypotheses $\left\{\Psi_{u}(k-1) \mid u=1,2, \ldots, N_{h}(k-1)\right\}$ and new association events $\left\{\psi_{i}(k) \mid i=0,1, \ldots, N_{m}(k)\right\}$ as

$$
\begin{aligned}
f\left\{\mathbf{x}(k) \mid \mathbf{Z}^{k}\right\}= & \sum_{u=1}^{N_{h}(k-1)} \sum_{i=0}^{N_{m}(k)} f\left\{\mathbf{x}(k) \mid \mathbf{Z}^{k}, \psi_{i}(k), \Psi_{u}(k-1)\right\} \\
& \cdot P\left\{\psi_{i}(k), \Psi_{u}(k-1) \mid \mathbf{Z}^{k}\right\} .
\end{aligned}
$$

Due to the conditioning on the association event $\psi_{i}(k)$ (which prescribes which measurement in $\mathbf{Z}_{k}$, if any, was target originated), the Gaussian pdf $f\left\{\mathbf{x}(k) \mid \mathbf{Z}^{k}, \psi_{i}(k), \Psi_{u}(k-1)\right\}$ can be calculated from the Gaussian pdf $f\left\{\mathbf{x}(k) \mid \mathbf{Z}^{k-1}, \mathbf{\Psi}_{u}(k-1)\right\}$ using the standard Kalman filter update equations, and again the posterior density will remain Gaussian. Therefore, assuming that the prior pdf is a Gaussian mixture, the posterior pdf $f\left\{\mathbf{x}(k) \mid \mathbf{Z}^{k}\right\}$ will also be a Gaussian 
mixture. The double summation of the preceding equation can be combined for convenience into a single summation over an equivalent set of indices. Defining a new set of association history hypotheses $\left\{\boldsymbol{\Psi}_{u^{\prime}}(k) \mid u^{\prime}=1,2, \ldots, N_{h}(k)\right\}$, where $N_{h}(k)=\left[N_{h}(k-1)\right]$ - $\left[N_{m}(k)+1\right]$, the double sum result of the preceding equation can be written as

$$
f\left\{\mathbf{x}(k) \mid \mathbf{Z}^{k}\right\}=\sum_{u^{\prime}=1}^{N_{h}(k)} f\left\{\mathbf{x}(k) \mid \mathbf{Z}^{k}, \Psi_{u^{\prime}}(k)\right\} P\left\{\Psi_{u^{\prime}}(k) \mid \mathbf{Z}^{k}\right\}
$$

where

$$
\begin{gathered}
f\left\{\mathbf{x}(k) \mid \mathbf{Z}^{k}, \Psi_{u^{\prime}}(k)\right\} \\
=f\left\{\mathbf{x}(k) \mid \mathbf{Z}^{k}, \psi_{i}(k), \Psi_{u}(k-1)\right\} \\
P\left\{\Psi_{u^{\prime}}(k) \mid \mathbf{Z}^{k}\right\}=P\left\{\psi_{i}(k), \Psi_{u}(k-1) \mid \mathbf{Z}^{k}\right\} .
\end{gathered}
$$

Note the Gaussian mixture form of this result. The association history event probabilities $P\left\{\boldsymbol{\Psi}_{u^{\prime}}(k) \mid \mathbf{Z}^{k}\right\}$ are commonly referred to as probability weights, and they are calculated using Bayes' rule (omitting unnecessary conditionings) as

$$
\begin{aligned}
P\left\{\boldsymbol{\Psi}_{u^{\prime}}(k) \mid \mathbf{Z}^{k}\right\} & \\
= & P\left\{\psi_{i}(k), \boldsymbol{\Psi}_{u}(k-1) \mid \mathbf{Z}^{k}\right\} \\
= & P\left\{\boldsymbol{\psi}_{i}(k), \boldsymbol{\Psi}_{u}(k-1) \mid \mathbf{Z}^{k-1}, \mathbf{Z}_{k}, N_{m}(k)\right\} \\
& P\left\{\mathbf{Z}_{k} \mid \boldsymbol{\psi}_{i}(k), \mathbf{\Psi}_{u}(k-1), \mathbf{Z}^{k-1}, N_{m}(k)\right\} \\
= & \frac{\cdot P\left\{\psi_{i}(k) \mid N_{m}(k)\right\} P\left\{\boldsymbol{\Psi}_{u}(k-1) \mid \mathbf{Z}^{k-1}\right\}}{P\left\{\mathbf{Z}_{k} \mid \mathbf{Z}^{k-1}, N_{m}(k)\right\}}
\end{aligned}
$$

where $P\left\{\boldsymbol{\Psi}_{u}(k-1) \mid \mathbf{Z}^{k-1}\right\}$ is the association history hypothesis probability from the previous processing cycle, and the remaining terms are evaluated according to the standard model.

The difficulty of data association is that every association history hypothesis from the previous processing cycle must be paired with every association event from the current set of measurements, and a new association history hypothesis must be created for each pairing. The challenge of tracking in the presence of clutter is clear from the expression:

$$
N_{h}(k)=\left[N_{h}(k-1)\right]\left[N_{m}(k)+1\right] .
$$

Thus, the number of components in the Gaussian mixture grows exponentially as new sets of measurements are received. It is therefore necessary to employ some method of reducing the number of components in the mixture while modifying the pdf surface as little as possible.

The ISE cost function $[27,28]$ provides a means of measuring the difference between two pdfs. Define the original mixture pdf of target state, containing $N_{h}(k)$ association history hypotheses, as $f\left\{\mathbf{x}(k) \mid \Omega_{N h}(k)\right\}$, where $\Omega_{N h}(k)$ represents the parameters of the $N_{h}(k)$ hypotheses derived from the measurements up to the current sample period $k$. The goal is thus to reduce these $N_{h}(k)$ hypotheses to a simplified representation, containing $N_{r}(k)$ hypotheses (the subscript $r$ denoting reduced), resulting in the simplified pdf $f\left\{\mathbf{x}(k) \mid \bar{\Omega}_{N r}(k)\right\}$, where $\bar{\Omega}_{N r}(k)$ represents the reduced set of parameters, containing, as closely as possible, the same information as the original set $\Omega_{N h}(k)$. To compare the two mixtures, the ISE algorithm simply subtracts the candidate pdf from the original pdf, squaring the result to rectify negative differences, and integrates to form the scalar:

$$
J_{S}=\int\left(f\left\{\mathbf{x}(k) \mid \boldsymbol{\Omega}_{N_{h}}(k)\right\}-f\left\{\mathbf{x}(k) \mid \bar{\Omega}_{N_{r}}(k)\right\}\right)^{2} d \mathbf{x}(k) .
$$

For the context of interest, in which the pdfs are sums of Gaussians, (7) can be evaluated without approximation or direct numerical integration [27, 28] as

$$
\begin{aligned}
J_{S}= & \int \sum_{i=1}^{N_{h}(k)} \sum_{j=1}^{N_{h}(k)} p_{i} \mathcal{N}\left\{\mathbf{X} ; \boldsymbol{\mu}_{i}, \mathbf{P}_{i}\right\} p_{j} \mathcal{N}\left\{\mathbf{X} ; \boldsymbol{\mu}_{j}, \mathbf{P}_{j}\right\} d \mathbf{X}(k) \\
& -2 \int \sum_{i=1}^{N_{h}(k)} \sum_{j=1}^{N_{r}(k)} p_{i} \mathcal{N}\left\{\mathbf{X} ; \boldsymbol{\mu}_{i}, \mathbf{P}_{i}\right\} \bar{p}_{j} \mathcal{N}\left\{\mathbf{X} ; \overline{\boldsymbol{\mu}}_{j}, \overline{\mathbf{P}}_{j}\right\} d \mathbf{X}(k) \\
& +\int \sum_{i=1}^{N_{r}(k)} \sum_{j=1}^{N_{r}(k)} \bar{p}_{i} \mathcal{N}\left\{\mathbf{X} ; \overline{\boldsymbol{\mu}}_{i}, \overline{\mathbf{P}}_{i}\right\} \bar{p}_{j} \mathcal{N}\left\{\mathbf{X} ; \overline{\boldsymbol{\mu}}_{j}, \overline{\mathbf{P}}_{j}\right\} d \mathbf{X}(k) \\
= & \sum_{i=1}^{N_{h}(k)} \sum_{j=1}^{N_{h}(k)} p_{i} p_{j} \mathcal{N}\left\{\boldsymbol{\mu}_{i} ; \boldsymbol{\mu}_{j}, \mathbf{P}_{i}+\mathbf{P}_{j}\right\} \\
& -2 \sum_{i=1}^{N_{h}(k)} \sum_{j=1}^{N_{r}(k)} p_{i} \bar{p}_{j} \mathcal{N}\left\{\boldsymbol{\mu}_{i} ; \overline{\boldsymbol{\mu}}_{j}, \mathbf{P}_{i}+\overline{\mathbf{P}}_{j}\right\} \\
& +\sum_{i=1}^{N_{r}(k)} \sum_{j=1}^{N_{r}(k)} \bar{p}_{i} \bar{p}_{j} \mathcal{N}\left\{\overline{\boldsymbol{\mu}}_{i} ; \overline{\boldsymbol{\mu}}_{j}, \overline{\mathbf{P}}_{i}+\overline{\mathbf{P}}_{j}\right\}
\end{aligned}
$$

where the basic terms within (8) are the multivariate normal (Gaussian) evaluations as a function of the first argument, with mean and covariance given by the two arguments after the semicolon.

To apply this cost function to form a mixture reduction algorithm, one must generate several candidate mixtures and select the reduced mixture with the lowest cost. Williams [27, 28] used an iterative greedy solver to find a reduced mixture with a prespecified number of components. Starting with the original mixture, it evaluates the cost of merging each pair of remaining components, and the cost of deleting each remaining component, and selects the lowest cost action. When merging components, it assigns parameters to keep the mean and covariance 
of the overall mixture unchanged:

$$
\begin{aligned}
& \text { Weight: } p_{c}=p_{1}+p_{2} \\
& \text { Mean: } \quad \boldsymbol{\mu}_{c}=\frac{1}{p_{1}+p_{2}}\left\{p_{1} \hat{\mathbf{x}}_{1}+p_{2} \hat{\mathbf{x}}_{2}\right\}
\end{aligned}
$$

Covariance: $\quad \mathbf{P}_{c}=\frac{1}{p_{1}+p_{2}}\left\{p_{1} \mathbf{P}_{1}+p_{2} \mathbf{P}_{2}\right.$

$$
\left.+\frac{p_{1} p_{2}}{p_{1}+p_{2}}\left(\hat{\mathbf{x}}_{1}-\hat{\mathbf{x}}_{2}\right)\left(\hat{\mathbf{x}}_{1}-\hat{\mathbf{x}}_{2}\right)^{\mathrm{T}}\right\} .
$$

The process is repeated until the desired number of components has been reached. The ISE algorithm has a major performance advantage over previous reduction methods in that it evaluates the impact of a merge or a prune on the entire original mixture at each time step. In testing [8], [9], [27], [28], the algorithm was shown to outperform all other algorithms by a substantial margin in heavy clutter when enough components $\left(N_{r}>15\right)$ were used to create an adequate fidelity representation of the target state pdf (e.g, a factor of two increase in average track life over the previously best algorithm [18-21] with $\left.N_{r}=35\right)$. However, the combinatoric nature of the comparisons based on (8) causes significant computational expense; see [28] for full quantification of this expense relative to that of alternative algorithms. For example, when average track life was evaluated as a function of mean computation time required per time step rather than number of components retained [28], other algorithms were more competitive with, and in some instances even exceeded the performance of, the ISE-based technique. Reference [28] also develops several enhancements (particularly accelerated matrix inversions and use of cached values of costs and other components so that real-time evaluations are performed only when necessary) for increasing the computational efficiency of the ISE-based algorithm without sacrificing accuracy. In the Monte Carlo simulations accomplished herein, implementation of these routines reduced computation times from several hours to a few minutes with imperceptible impact on performance; the fully accelerated algorithms were used for all the trials of the performance analysis described in Section V. This algorithm can be considered similar to the iterative pairwise replacement algorithm [22] used by the nonparametric statistics community; the authors were not aware of this work until after the development of the ISE algorithm.

\section{MULTIPLE MODEL ADAPTATION TO TRAJECTORY VARIATIONS}

The preceding section accounts for tracking a single target in dense clutter, assuming that a valid depiction of target trajectories is adequately described by a single dynamics model. Of considerable concern

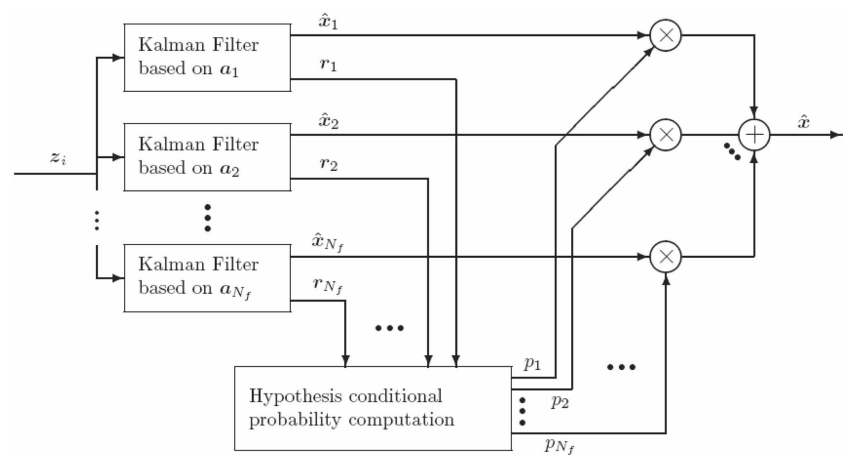

Fig. 1. MMAE algorithm.

is tracking a target that can exhibit substantially different trajectory characteristics over time, as due to the onset of jinking maneuvers. For such applications, no single model would generally be adequate. Multiple model approaches [2-5, 12] are ideally suited to this scenario and have been used with significant success in handling such unknown and/or strongly changing target trajectory attibutes.

Fig. 1 depicts a conventional MMAE algorithm $[10,12]$. Sensor measurements are presented to a bank of $N_{f}$ parallel Kalman filters, each based upon a different assumed (constant) model for target dynamics (represented by the parameter vector $\mathbf{a}$ assuming one of its possible values, $\mathbf{a}_{1}-\mathbf{a}_{N f}$ ). $N_{f}$ is the number of discrete values a might assume, and thus it is the number of filters in the parallel bank. Each filter produces its own state estimate $\hat{\mathbf{x}}_{m}(k \mid k-1)$ before the measurement update at time instant $k$ and the corresponding estimate $\hat{\mathbf{x}}_{m}(k \mid k)$ after that update, as well as its own residual at that time, $\mathbf{r}_{m}(k)$ :

$$
\mathbf{r}_{m}(k)=\mathbf{z}(k)-\mathbf{H} \hat{\mathbf{x}}_{m}(k \mid k-1)
$$

where $\mathbf{H}$ is the measurement matrix that relates measurements $\mathbf{z}(k)$ to the state variables $\mathbf{x}(k)$. These residuals are used to compute the hypothesis conditional probabilities $p_{m}(k)$ for $m=1,2, \ldots, N_{f}$, according to the iteration:

$$
p_{m}(k)=\frac{f\left\{\mathbf{z}(k) \mid \mathbf{a}_{m}, \mathbf{Z}(k-1)\right\} \cdot p_{m}(k-1)}{\sum_{j=1}^{N_{f}} f\left\{\mathbf{z}(k) \mid \mathbf{a}_{j}, \mathbf{Z}(k-1)\right\} \cdot p_{j}(k-1)} .
$$

Each of these probabilities can be interpreted as the conditional probability that the associated Kalman filter is the most appropriate elemental filter to be employing at the current time to describe the target dynamics. The conditional probability density for the incoming measurement in this iteration is given by

$$
\begin{aligned}
f\left\{\mathbf{z}(k) \mid \mathbf{a}_{m}, \mathbf{Z}(k-1)\right\} & =\frac{1}{(2 \pi)^{s / 2}\left|\mathbf{A}_{m}(k)\right|^{1 / 2}} \exp \{\cdot\} \\
\{\cdot\} & =\left\{-\frac{1}{2} \mathbf{r}_{m}^{\mathrm{T}}(k) \mathbf{A}_{m}^{-1}(k) \mathbf{r}_{m}(k)\right\}
\end{aligned}
$$


where $s$ is the dimension of the measurement, and $\mathbf{A}_{m}(k)$ is the filter-computed covariance for the measurement residual. If the hypothesized model within a particular elemental Kalman filter is a good representation of the real-world target trajectory, then the scalar quadratic form premultiplied by $[-1 / 2]$ in the exponent should be distributed as chi-squared with $s$ degrees of freedom, and have an expected value of $s$. Thus, in practice, if the hypothesized model is a good representation, the quadratic form should assume a value of about $s$. If the hypothesized model is incorrect, then this should assume a value different from $s$ and often more than an order of magnitude larger than $s$ since the residuals are larger than "anticipated" by the computed $\mathbf{A}_{m}(k)$, resulting in the corresponding $p_{m}(k-1)$ being premultiplied by a very small number in the iterative computation in (11).

As shown in Fig. 1, the MMAE produces an output state estimate as the probability-weighted average of the outputs of the individual elemental Kalman filters, along with a corresponding error covariance:

$$
\begin{aligned}
\hat{\mathbf{x}}(k \mid k)= & \sum_{m=1}^{N_{f}} \hat{\mathbf{x}}_{m}(k \mid k) p_{m}(k) \\
\mathbf{P}(k \mid k)= & \sum_{m=1}^{N_{f}} p_{m}(k) \\
& \cdot\left\{\mathbf{P}_{m}(k \mid k)+\left[\hat{\mathbf{x}}_{m}(k \mid k)-\hat{\mathbf{x}}(k \mid k)\right]\right. \\
& \left.\cdot\left[\hat{\mathbf{x}}_{m}(k \mid k)-\hat{\mathbf{x}}(k \mid k)\right]^{\mathrm{T}}\right\} .
\end{aligned}
$$

Note that, in the iteration to compute $p_{m}(k)$ given by $(11)$, if $p_{m}(k-1)$ is ever computed as zero, that $p_{m}$ will be zero thereafter, even if the associated model becomes the best model to represent the real world (a phenomenon called "lockout"). To handle the possibility of time-varying parameters in the real world with an MMAE and to preclude lockout, it is important to effect a lower bound on the probabilities computed by the $p_{m}(k)$ iteration. For this research, a lower bound of $10^{-3}$ was used. Moreover, if a specific elemental filter diverges (as exhibited by $\left[\mathbf{r}_{m}^{\mathrm{T}}(k) \mathbf{A}_{m}^{-1}(k) \mathbf{r}_{m}(k)\right]$ taking on a value much greater than $s$ ), that filter can and should be restarted with the MMAE state estimate (computed without the divergent filter or filters).

The MMAE algorithm is based on the assumption that the parameter vector is an unknown constant. If instead it is modeled as the output of a stochastic process, one can use the IMM algorithm [3-5, 7, 14]. The $p_{m}(k)$ iteration already shown can be considered a measurement update algorithm, and a Markov model for the vector of such $p_{m}(k) \mathrm{s}$ is considered to be a time propagation model. If the Markov probability state transition matrix were assumed to be an identity matrix, then the corresponding IMM would be equivalent to an MMAE without restarts. In many designs, this matrix is formed as a small perturbation from an identity matrix, allowing transitions from one mode (parameter value; assumed model) to another over a propagation sample period. Thus, for an MMAE, each elemental filter's state estimate is the output of a propagation by that filter's dynamics model only (consistent with the static parameter assumption). In an IMM on the other hand, each filter's estimate is the output of a weighted history of propagations based on all filters in the system (consistent with the assumption of switching modes or parameter values). At the end of every mixing cycle, one calculates the mixed estimates and a posteriori modal probabilities for recursion into the next cycle. The mixed estimates can be calculated as

$$
\begin{aligned}
\hat{\mathbf{x}}^{i}(k \mid k)= & \sum_{j=1}^{N_{f}} P\left\{M_{k, j} \mid M_{k+1, i}, \mathbf{Z}^{k}\right\} \hat{\mathbf{x}}_{j}(k \mid k) \\
\mathbf{P}^{i}(k \mid k)= & \sum_{j=1}^{N_{f}} P\left\{M_{k, j} \mid M_{k+1, i}, \mathbf{Z}^{k}\right\} \\
& \cdot\left\{\mathbf{P}_{j}(k \mid k)+\left[\hat{\mathbf{x}}_{j}(k \mid k)-\hat{\mathbf{x}}^{i}(k \mid k)\right]\right. \\
& \left.\cdot\left[\hat{\mathbf{x}}_{j}(k \mid k)-\hat{\mathbf{x}}^{i}(k \mid k)\right]^{\mathrm{T}}\right\} .
\end{aligned}
$$

where $M_{k, j}$ represents the hypothesis that mode $j$ is assumed at time instant $k$, and the mixing probabilities are computed as

$$
P\left\{M_{k, j} \mid M_{k+1, i}, \mathbf{Z}^{k}\right\}=\frac{\mathbf{T}_{i j} P\left\{M_{k, j} \mid \mathbf{Z}^{k}\right\}}{\sum_{n=1}^{N_{f}} \mathbf{T}_{i n} P\left\{M_{k, n} \mid \mathbf{Z}^{k}\right\}}
$$

where $\mathbf{T}_{i j}$ is the $i-j$ component of the Markov probability state transition matrix. The final modal probabilities after mixing are given by:

$$
\begin{aligned}
P\left\{M_{k, j} \mid \mathbf{Z}^{k}\right\} & \\
& f\left\{\mathbf{z}(k) \mid M_{k, j}, \mathbf{Z}^{k-1}\right\} \\
= & \frac{\cdot \sum_{i=1}^{N_{f}} P\left\{M_{k, j} \mid M_{k-1, i}\right\} P\left\{M_{k-1, i} \mid \mathbf{Z}^{k-1}\right\}}{\sum_{n=1}^{N_{f}} f\left\{\mathbf{z}(k) \mid M_{k, n}, \mathbf{Z}^{k-1}\right\} P\left\{M_{k, n} \mid \mathbf{Z}^{k-1}\right\}} .
\end{aligned}
$$

From a designer's perspective, the difference between an MMAE and an IMM is this: an MMAE requires specification of a lower bound for computed probabilities in order to handle changing parameter values. In contrast, an IMM requires specification of all the entries in the Markov model probability state transition matrix, which is often accomplished in a rather ad hoc manner, to handle such changes. In most applications, it is fundamentally easier to accomplish the former than the latter, particularly if iterative tunings are required. 


\section{COMBINED ALGORITHM ARCHITECTURES}

One potentially useful algorithm architecture $[13,24]$ would replace each Kalman filter within a conventional MMAE or IMM with an ISE-based algorithm (able to handle clutter) that assumes the adequacy of the same particular dynamics model and discretized parameter choice (i.e., "mode"). To accomplish the one-for-one replacement of Kalman filters with ISE-based algorithms, each such ISE-based algorithm based on mode $m$ must be able to produce an "equivalent" residual $\mathbf{r}_{m}(k)$ and associated residual covariance $\mathbf{A}_{m}(k)$, since the probability calculations within either the MMAE or IMM depend on evaluating the scalar $\left[\mathbf{r}_{m}^{\mathrm{T}}(k) \mathbf{A}_{m}^{-1}(k) \mathbf{r}_{m}(k)\right]$, as seen in (12). The equivalent residual $\mathbf{r}_{m}(k)$ can be written as a probability-weighted sum, using the residuals $\mathbf{r}_{m, u}(k)$ from each of the individual Kalman filters based on the assumption that dynamics model (mode) $m$ and association history hypothesis $u$ are correct:

$$
\begin{aligned}
& \hat{\mathbf{r}}_{m}(k)= \sum_{n=1}^{N_{h}(k)} \hat{\mathbf{r}}_{m, u}(k) \cdot P\left\{\boldsymbol{\Psi}_{u}(k) \mid \mathbf{Z}^{k}\right\} \\
& \mathbf{A}_{m}(k)= \sum_{u=1}^{N_{h}(k)}\left\{\mathbf{A}_{m, u}(k)+\left[\hat{\mathbf{r}}_{m, u}(k)-\hat{\mathbf{r}}_{m}(k)\right]\right. \\
&\left.\cdot\left[\hat{\mathbf{r}}_{m, u}(k)-\hat{\mathbf{r}}_{m}(k)\right]^{\mathrm{T}}\right\} \\
& \\
& \cdot P\left\{\boldsymbol{\Psi}_{u}(k) \mid \mathbf{Z}^{k}\right\} .
\end{aligned}
$$

Measurement gating is an important issue. MHT filters typically use measurement gates to preclude the need to process all the measurements in the measurement space. A measurement gate is formed around the predicted target location for each component in the Gaussian mixture, so each entire ISE-based algorithm (viewed as an entity) uses a "union" of the separate gates associated with each of its mixture components. A designer may or may not want to form the gate for each component's Kalman filter as the true union of all component filter gates, rather than just that one component filter's locally computed gate-a tradeoff analysis is needed and designers differ in how they treat this issue. There is also a need to "union" gates among the component ISE algorithms within an MMAE or IMM. This is necessitated by the fact that multiple model algorithm relationships are all conditioned on the same measurement history being presented to each elemental filter.

Initial performance analyses [13, 24] indicated the extreme importance of proper gating for maximum tracking benefit. Investigating this essential issue in fact led to the second and superior algorithm architecture $[8,9,25]$, in the form of an ISE-based algorithm of MMAEs (or IMMs), rather than an MMAE (or IMM) of ISE-based filters. In such an architecture, it is clear that gating should be performed such that each MMAE or IMM is separately gated so that the same measurements are provided to all of the Kalman filters within that MMAE or IMM-in a well-tuned algorithm, that does not require a unioning of individual gates but is simply the gate associated with the elemental Kalman filter based on the most aggressive dynamics model.

Thus, four architectures emerged, 1) an MMAE of ISE-based algorithms, 2) an IMM of ISE-based algorithms, 3) an ISE-based algorithm of MMAEs, and 4) an ISE-based algorithm of IMMs. Of these, 3 and 4 have proven superior to 1 and 2, in part due to the more effective gating accomplishable for algorithms 3 and 4. Between these two highest ranking architectures, 3 has been shown to provide the best performance of all, as seen in the next section.

One might also consider expanding the Gaussian mixture at each time step due to the possible mode changes as well as data association uncertainties, and then applying the mixture reduction algorithm (MRA). This would avoid introducing the MMAE or IMM techniques altogether. However, this significantly increases the number of mixture components to be handled by the reduction algorithm, severely complicates effective gating strategies and other aspects of tracker operation, and demonstrated inferior performance to that of the four architectures of the preceding paragraph in preliminary investigations. Therefore, only those four algorithm architectures are pursued in the next section.

\section{PERFORMANCE EVALUATION}

The four proposed tracker architectures were tested via computer simulation against realistic flight data. Ten minutes of flight data were recorded at $2 \mathrm{~Hz}$ using FlightGear [15], an accurate open-source flight simulator based on a 6-degree-of-freedom flight dynamics model. Because multiple model structures are inherently better at identifying well-separated maneuver modes, an agile aircraft model was selected: the General Dynamics F-16 fighter found in many military inventories. A $175 \mathrm{~s}$ subset of the flight data displaying a representative spectrum of flight modes was selected for performance evaluation to reduce computation time. See Fig. 2. The recorded positions were projected onto a two-dimensional plane by discarding a single axis (the east axis), creating an orthographic projection representative of a radar observing the target from an eastward position. To simulate measurement noise, the recorded positions were corrupted with discrete-time white Gaussian noise of variance $R=100 \mathrm{~m}^{2}$.

All filters were based on the constant-acceleration flight dynamics model, a special case of a first-order Gauss-Markov acceleration (FOGMA) in which the correlation time has become infinite, which is shown 


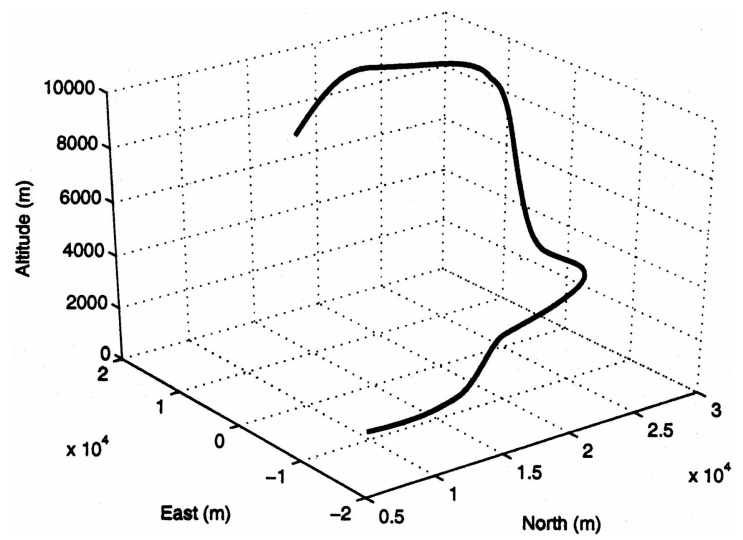

Fig. 2. Real target flight segment.

here in equivalent discrete-time form [11]:

$$
\begin{aligned}
\mathbf{x}(k)= & {\left[\begin{array}{c}
p_{x}(k) \\
v_{x}(k) \\
a_{x}(k) \\
p_{y}(k) \\
v_{y}(k) \\
a_{y}(k)
\end{array}\right]=\left[\begin{array}{cccccc}
1 & T & \frac{T^{2}}{2} & 0 & 0 & 0 \\
0 & 1 & T & 0 & 0 & 0 \\
0 & 0 & 1 & 0 & 0 & 0 \\
0 & 0 & 0 & 1 & T & \frac{T^{2}}{2} \\
0 & 0 & 0 & 0 & 1 & T \\
0 & 0 & 0 & 0 & 0 & 1
\end{array}\right] \mathbf{x}(k-1) } \\
& +\left[\begin{array}{cc}
\frac{T^{2}}{2} & 0 \\
T & 0 \\
1 & 0 \\
0 & \frac{T^{2}}{2} \\
0 & T \\
0 & 1
\end{array}\right] \mathbf{w}(k-1)
\end{aligned}
$$

where $T$ is the sample period, and $\mathbf{w}(k)$ is a discrete-time zero-mean white noise process such that

$$
E\left\{\mathbf{w}(k) \mathbf{w}(k)^{\mathrm{T}}\right\}=\mathbf{Q}=q \mathbf{I} .
$$

The clutter density was set at $\lambda=.0001 \mathrm{pts} / \mathrm{m}^{2}$, yielding 400 expected clutter points within the clutter region per epoch. This clutter density was selected so that the Monte Carlo simulations would lose track in approximately $10 \%$ of the runs, indicating that the ISE reduction algorithm is running near the peak of its performance capability for the given maneuver level and clutter density. Clutter was generated according to a Poisson distribution within a square region with sides $200 R^{1 / 2}$ centered on the tracker's best estimate of the target location. The probability of detection $P_{d}$ was set to unity such that one of the measurements was always target originated. The gate size was set such that the probability of a target-originated measurement being in the association gate was $P_{g}=$ 0.99 . On average, about 6 clutter points fell within the acceptance gate (but varying from about 2 for very benign conditions to the teens for very aggressive maneuvering), yielding an average of just over 100 Gaussian mixture components before reduction (but varying from about 30 to over 200), in accordance with (6), to be driven to 15 components by the MRA described at the end of Section II.

Fifty Monte Carlo simulations were performed for each tracker configuration, with each simulation run for 350 epochs or until track loss was declared. Track loss is simply defined as five consecutive epochs without the target-originated measurement falling within an association gate for any of the mixture hypotheses. The Monte Carlo sample statistics are calculated using only simulation data from nondivergent simulations, while also monitoring the number of simulations that yield track loss.

The multiple model configurations were composed of two filters, one assuming benign dynamics $\left(0.5 \mathrm{~m} / \mathrm{s}^{3}\right.$ jerk $)$, and another assuming aggressive dynamics $\left(4 \mathrm{~m} / \mathrm{s}^{3}\right)$. In simulations using MMAEs, the lower bound was set to 0.001 , and restarts for divergent filters were initiated when the Gaussian pdf of the target predicted position evaluated at the incoming measurement (see (12)) was less than $1 \times 10^{-5}$. For the IMM test cases, the Markov probability state transition matrix was constructed as a diagonally dominant matrix with "leakage" terms on the off-diagonal:

$$
\mathbf{T}(k \mid k-1)=\left[\begin{array}{ll}
0.9 & 0.1 \\
0.1 & 0.9
\end{array}\right] .
$$

Fig. 3 shows mean \pm one standard deviation flow of elemental filter probability at each epoch (for those Monte Carlo simulations that have not lost track), both with and without clutter (using the ISE-based MHT of MMAEs for the clutter case and the corresponding MMAE for the clutter-free case). The magnitude of the aircraft jerk is shown below each and indicates that the multiple model structure is correctly identifying the appropriate maneuver mode for the target's level of dynamics at any given time. Moreover, note that the presence of clutter does not significantly impede the probability flow of the tracker. The impact due to clutter can be seen as higher variance in the probability flow. Note the characteristic rapid flow of probability to the aggressive filter at maneuver onset (e.g., at epoch 200) and slow return to the benign filter after maneuver (e.g., 80-150), caused by the larger value of $\mathbf{A}_{m}(k)$ in (12) for the aggressive dynamics model than for the benign model.

Fig. 4 exhibits a transition from benign to aggressive flight maneuvering for a single Monte Carlo run in which the true dynamics are gradually becoming more aggressive. The probability flow for the benign filter is shown for both a cluttered and a noncluttered case. Note the corruption caused by the clutter in the benign and transition regions-even when the maneuver dynamics are distinctly benign, 

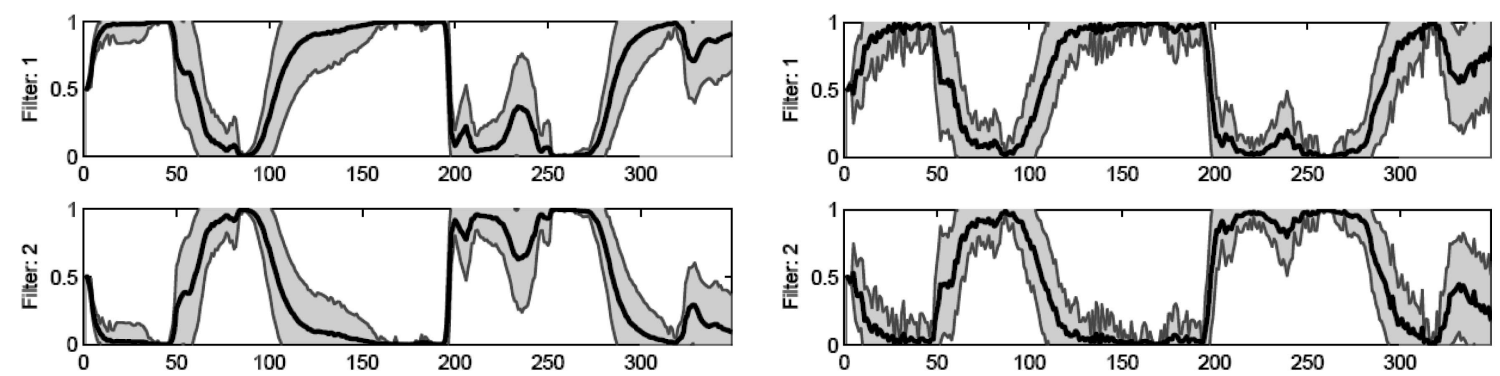

(a)

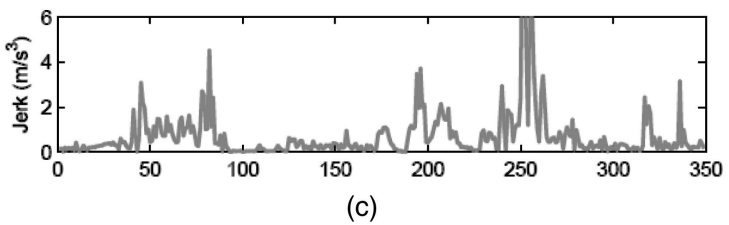

(b)

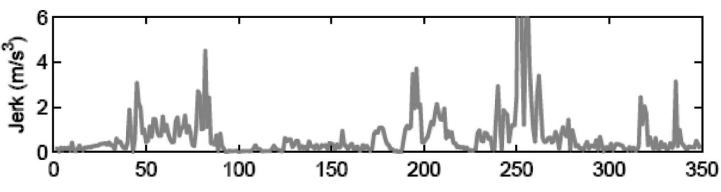

(d)

Fig. 3. Comparison of MMAE elemental filter flow with and without clutter. Filter 1 based on a benign dynamics model and filter 2 based on an aggressive dynamics model. (a) MMAE probability $\pm \sigma$ flow without clutter. (b) MMAE probability $\pm \sigma$ flow with clutter. (c) Magnitude of F-16 jerk. (d) Magnitude of F-16 jerk.

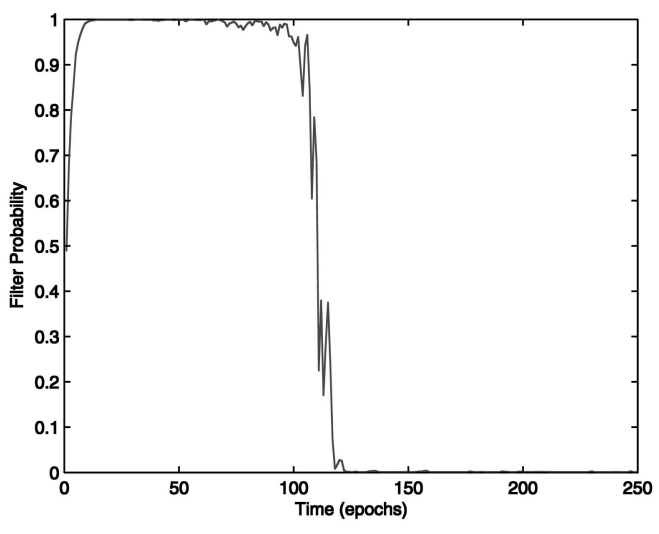

(a)

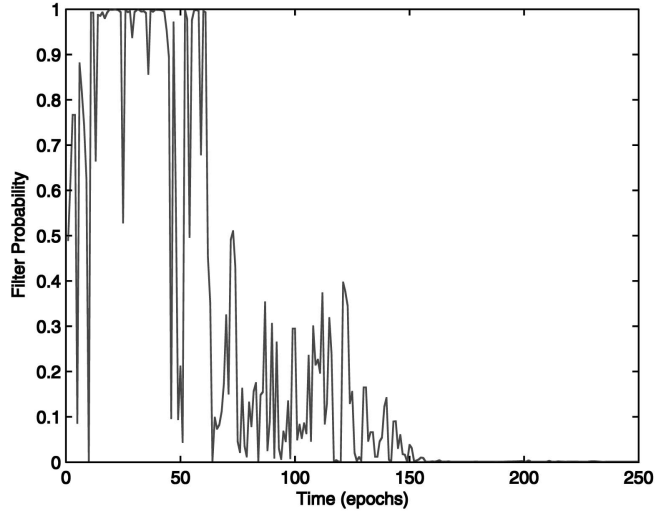

(b)

Fig. 4. Effect of clutter on multiple model probability flow. (a) Benign filter probability flow without clutter. (b) Benign filter probability flow with clutter.

clutter can cause residuals in the aggressively tuned filter to appear more favorable in the computations of (11) and (12). In these cases, the filter flow is not a distinctive transfer of operation to the appropriate filter, but rather is a time-averaged dominance of the appropriate filter. This effect is not apparent in the aggressive modes, for which the flow is distinctive and appropriate. Consequently, the benefit of the benign filters in clutter is somewhat reduced compared with that of the clutter-free case.

Figs. 5-7 display the tracking characteristics of an ISE-based MHT algorithm of Kalman filters based on the aggressive dynamics models, an ISE-based MHT of MMAEs, and an ISE-based MHT of IMMs, respectively. The MMAEs and IMMs were composed of elemental filters based on the benign and aggressive dynamics models as described earlier. The benign filter was incapable of tracking the aircraft maneuvering in clutter (over $90 \%$ of the runs lost track between 100 and 180 samples into the simulatons), so no meaningful statistics could be collected on an ISE-based MHT of Kalman filters based on that benign model.

In each figure, the $x$ (azimuth) and $y$ (elevation) position and velocity are shown as the top four plots. The Monte Carlo sample mean errors \pm two standard deviations $(2 \sigma)$ are shown as solid black lines, and the filter-computed standard deviations $\left(0 \pm 2 \sigma_{\text {filter }}\right)$ are overlayed in gray. For a well-tuned filter, the actual and computed values should agree, as can be seen for the majority of time in the plots. Dashed gray lines show the corresponding $\left(0 \pm 2 \sigma_{\text {filter }}\right)$ based only on the aggressive-model Kalman filter-these are obvious only in the second row of plots in Fig. 6, but are actually present in all four top plots in Figs. 5-7. Where the solid gray lines are substantially narrower than the dashed, the probability weighting is heavily on the benign elemental filter, and where the solid gray lines are significantly wider than the dashed indicates where the mean 

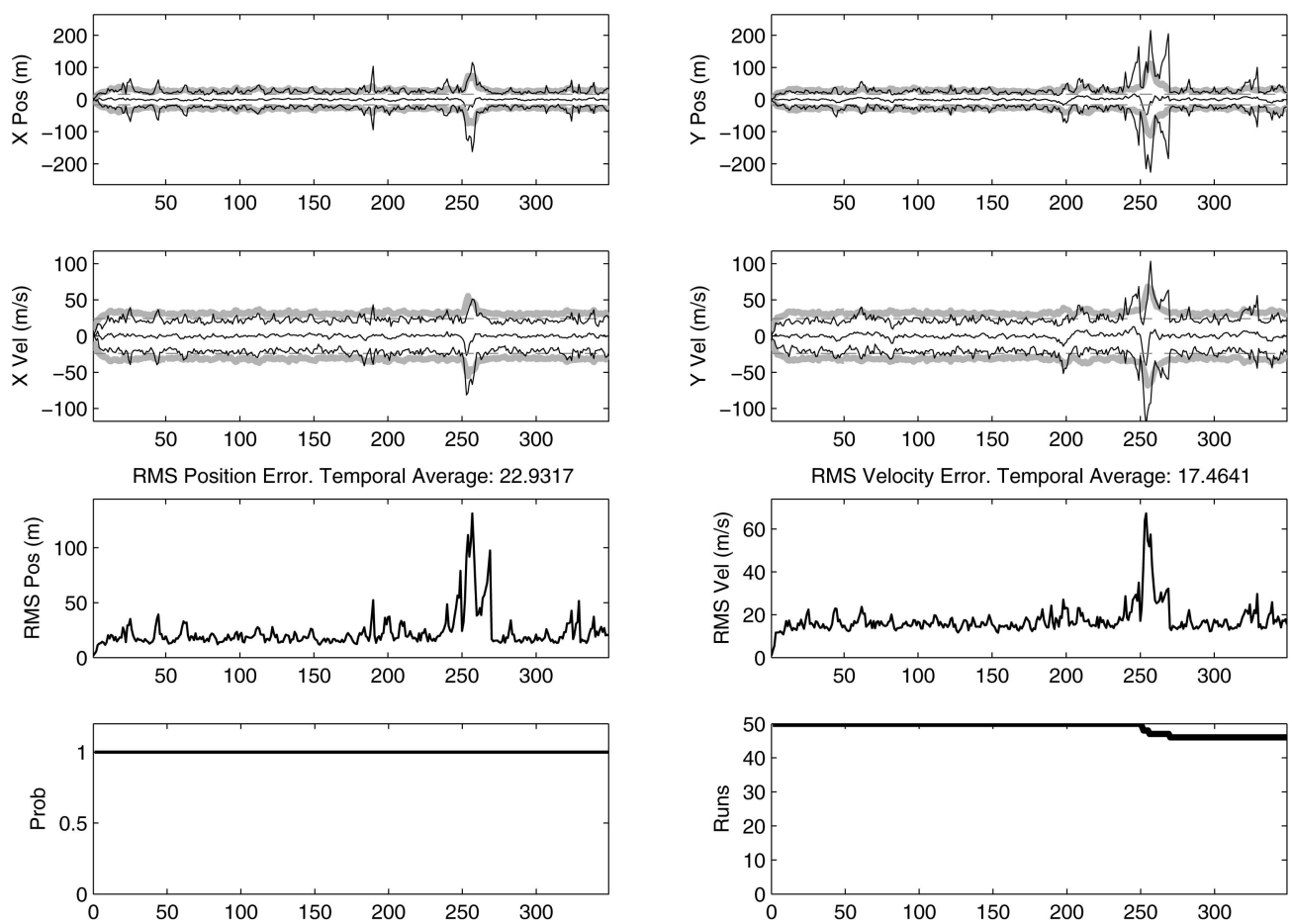

Fig. 5. Performance of ISE-based MHT of Kalman filters based on aggressive dynamics model.
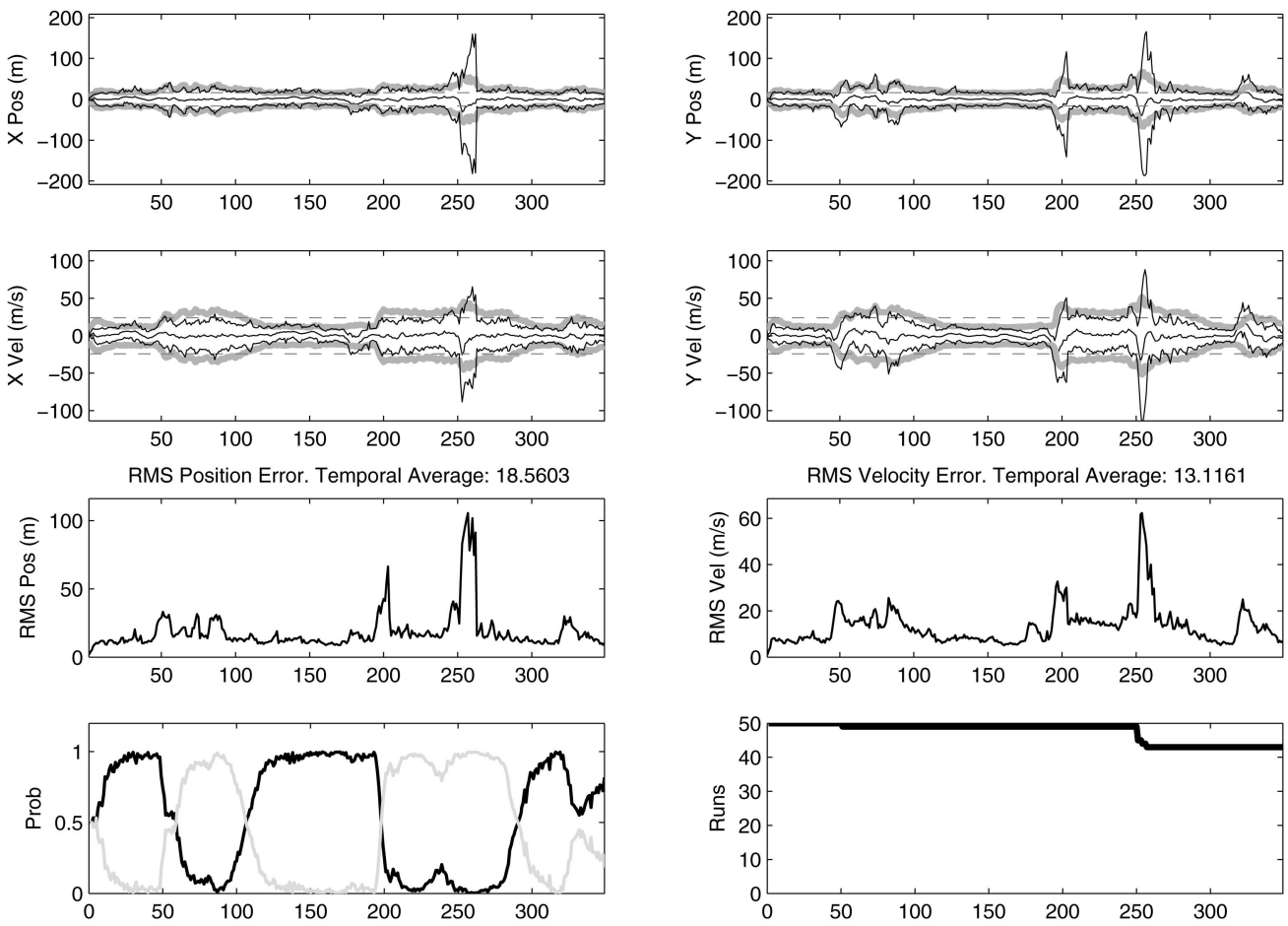

Fig. 6. Performance of ISE-based MHT of MMAEs.

spreading terms in computations like (13) are dominating.

The third row of plots in each figure depict the rms overall position and velocity errors versus time. The bottom left plot shows the mean probability assigned to each of the two dynamics models, and the bottom right plot indicates the number of Monte Carlo runs in which track has not been lost.
In all three figures, the first maneuvers from epochs 45-100 are tracked appropriately, as can be seen from the zero-mean error. Furthermore, in Fig. 6, the probability flow for the MMAE is apparent in the reduced computed and actual filter standard deviations, as compared with those of Fig. 5. The probability flow for the IMM (Fig. 7) is less definitive, as is expected due to the model mixing that 

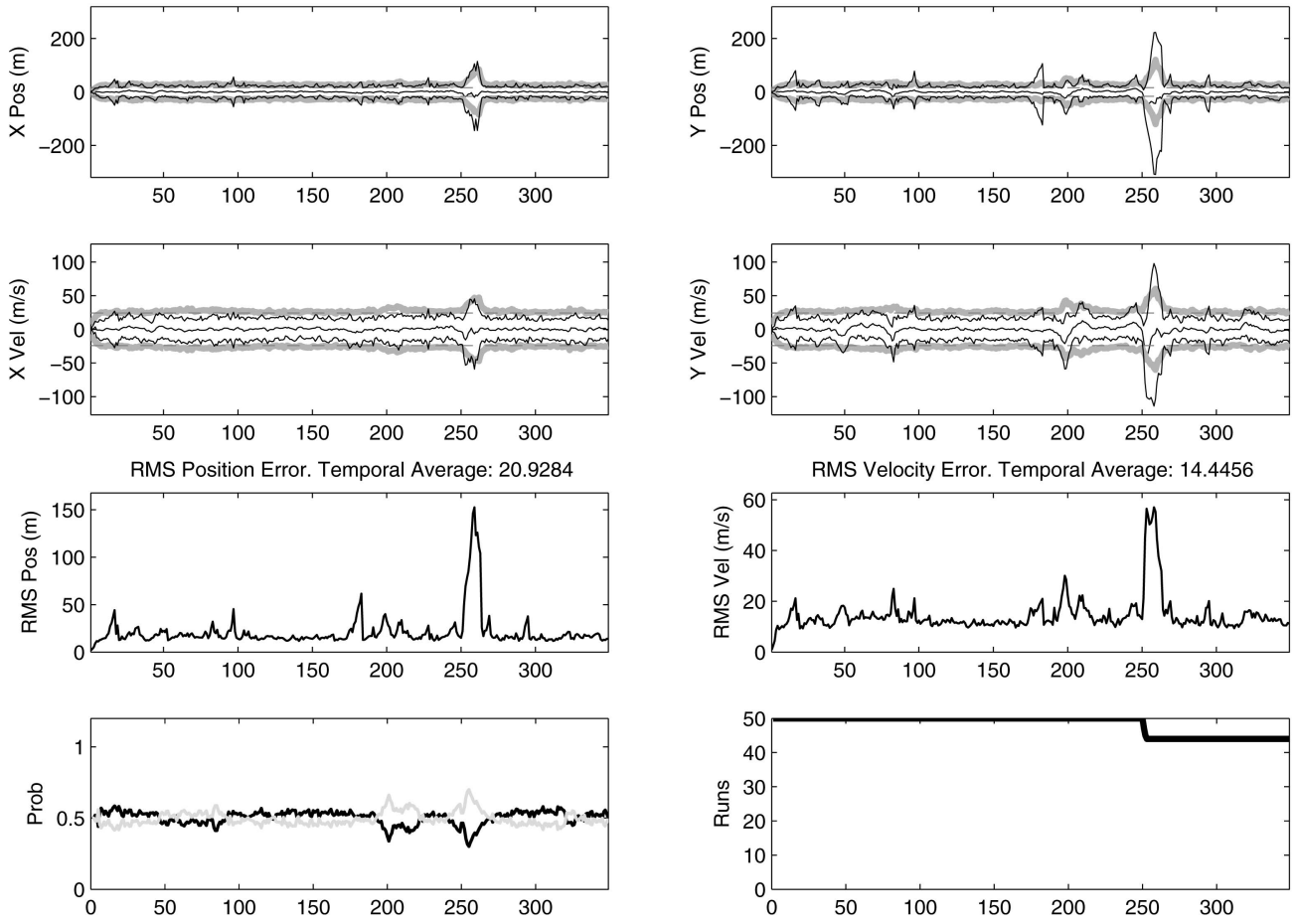

Fig. 7. Performance of ISE-based MHT of IMMs.

occurs over time in the IMM-but the benefit during the benign portions of flight is also significantly less than that of the MMAE. The second set of more aggressive actual maneuvers, epochs 200-250, exhibit considerable offset in the mean error, which did not exist in the comparable uncluttered case (see [8]), indicating that the clutter is affecting the algorithm's ability to track the target. This can also be seen in the spikes in the actual and filter-computed standard deviations around epoch 250. These spikes are caused by the mean spreading terms in the Gaussian mixture computations analogous to (13) and indicate that the hypotheses that compose the mixture are no longer coincident in parameter space. These inevitable periods of deferred decision-making are an inherent part of the MHT process, but they also introduce significant estimation errors, as can be seen in the manifested offset. The plots show the mean errors of 50 Monte Carlo simulations, but observation of single runs indicates that the deferred decision errors can be up to $500 \mathrm{~m}$ in magnitude. It was also observed that the multiple model structures tended to increase the magnitude of these errors slightly.

Table I shows the temporally averaged rms errors for the filter configurations depicted in Figs. 5-7 and also for the alternative architectures of an MMAE or IMM of ISE-based algorithms. Again, the ISE of Kalman filters based on the benign model is not shown because it is incapable of tracking the target once maneuvers are initiated. To set a context for these values, the conventional MMAE based on the same two elemental filters achieved an rms position
TABLE I

Temporally Averaged rms Position and Velocity Errors for Different Configurations

\begin{tabular}{lccccc}
\hline \hline & $\begin{array}{c}\text { ISE } \\
\text { of } \\
\text { Aggs }\end{array}$ & $\begin{array}{c}\text { ISE } \\
\text { of } \\
\text { MMAEs }\end{array}$ & $\begin{array}{c}\text { ISE } \\
\text { of } \\
\text { IMMs }\end{array}$ & $\begin{array}{c}\text { MMAE } \\
\text { of } \\
\text { ISEs }\end{array}$ & $\begin{array}{c}\text { IMM } \\
\text { of } \\
\text { ISEs }\end{array}$ \\
\hline $\begin{array}{l}\text { rms Position Error (m) } \\
\text { rms Velocity Error } \\
(\mathrm{m} / \mathrm{s})\end{array}$ & 22.9 & 18.6 & 20.9 & 22.7 & 26.6 \\
$\begin{array}{l}\text { Benign rms Position } \\
\quad \text { Error (m) }\end{array}$ & 18.1 & 11.9 & 14.4 & 16.7 & 15.4 \\
$\begin{array}{l}\text { Benign rms Velocity } \\
\quad \text { Error (m/s) }\end{array}$ & 15.4 & 7.8 & 11.6 & 13.4 & 12.4 \\
\hline
\end{tabular}

Note: Average taken over entire simulation and over only partial region of benign flight.

error of $10.6 \mathrm{~m}$ and an rms velocity error of $10.5 \mathrm{~m} / \mathrm{s}$ in clutter-free simulations.

In clutter, the multiple model structures should have about the same performance as the ISE-based algorithm of only aggressive-model Kalman filters while the aircraft is in an aggressive maneuver mode (as seen in Table I itself, as well as in the upper plots of Figs. 5-7, and also in the bottom right plots that show essentially the same number of cases, about $10 \%$, in which loss-of-track occurred). However, multiple model structures ought to have better tracking fidelity during the benign portions of the flight than just an aggressive-mode Kalman filter. Consequently, four values are shown for each architecture in Table I, for the position and velocity errors for both the entire flight and for a benign segment in which the aircraft was in straight-and-level flight. As expected, the 
performance during the benign periods is significantly better for all the multiple model structures compared with the single model structure in both position and velocity estimates. The same improvement is apparent for the entire duration, but the magnitude of improvement is smaller because the averages include periods in which the multiple model and single filters are operating with identical parameters.

From Table I, it can also be seen by comparing the fourth and fifth columns to the second and third columns, that an ISE-based algorithm of MMAEs or IMMs significantly outperforms the corresponding MMAE or IMM of ISE-based algorithms. Moreover, the algorithms incorporating MMAE structures consistently outperform the analogous architecture but based on an IMM.

Now focus on the first three columns of Table I. The ISE-based MHT of MMAEs shows a 19\% reduction in rms position error compared with an ISE-based MHT of aggressive-mode Kalman filters when averaging is performed over the entire simulation, and a $34 \%$ reduction when averaging over only the partial region of benign flight. In comparison, the ISE-based MHT of IMMs shows only $9 \%$ and $17 \%$ reductions, respectively. With respect to $\mathrm{rms}$ velocity errors, the ISE-based MHT of MMAEs exhibits a $25 \%$ reduction over the entire simulation and a $49 \%$ reduction over the region of benign flight, whereas the ISE-based MHT of IMMs yields only $18 \%$ and $25 \%$ reductions, respectively. A purposely fair head-to-head comparison between MMAE and IMM algorithms was conducted, using very reasonable choices for the MMAE lower bound on computed probabilities (0.001) and for the IMM Markov model probability state transition matrix (see (20)). Some tuning on these parameters was performed, but these final choices are representative of best achievable performance for each algorithm for this simulation environment. One might have anticipated better performance from the IMM than from the MMAE, especially since there are numerous practical examples of good performance from an IMM, but as seen above, instead the MMAE consistently outperformed the IMM by a considerable margin.

The underlying reason for this discrepancy in performance can be seen from Figs. 6 and 7. The MMAE maintains completely separated benign-mode and aggressive-mode elemental Kalman filters, and the relative adequacy of the two model assumptions is quite distinguishable, as evidenced by the strong probability flows in the lower left plot of Fig. 6 when the actual target changes its strength of maneuvering. In contrast, the intermixing within the IMM causes the residuals from its elemental filters to be less distinguishable from each other, and the probability hovers around $50 \%$ for each one in the lower left plot of Fig. 7: they are virtually indistinguishable from one another. Therefore, when the target actually exhibits benign trajectory motion, there is considerable payoff for the MMAE heavily weighting the benign-mode Kalman filter (as seen in the lower left plot of Fig. 6). The performance benefit can be seen in the corresponding necking down of the upper four plots of error mean \pm one standard deviation in Fig. 6 during that time; this is most readily seen in the velocity plots, the second row of plots. In contradistinction, the IMM does not exhibit either substantial probability flow or the associated beneficial necking down of the mean $\pm 1 \sigma$ plots in Fig. 7. Since the probabilities are hovering at about $50 \%$ for each elemental filter, each is receiving very similar initial conditions at each mixing cycle, and so the distinguishability of the two hypothesized modes is confined to differences they cause over only a single propagation cycle (before intermixing occurs again at the next update cycle). The MMAE, on the other hand, allows the difference between benign and aggressive models to be seen more persistently in the outputs and residuals of the elemental filters, thereby providing better model distinguishability and consequent performance improvement in this application.

Of the four proposed algorithm architectures, the ISE-based MHT of MMAEs has been shown to be the most capable. Furthermore, it outperforms an ISE-based algorithm with only Kalman filters based on the aggressive dynamics model by a wide margin.

\section{CONCLUSION}

Four architectures for a tracker that combines an ISE-based reduced Gaussian mixture MHT (to handle clutter) and either an MMAE or IMM (to handle time-varying trajectory attributes, as due to maneuver onset) have been evaluated. Of these, the ISE-based MHT of MMAEs has been shown to be superior in a simulation of an F-16 in dense clutter exhibiting benign flight, high-G time-correlated turns, and sudden jinking maneuvers.

The four tracking algorithms were capable of maintaining track on the target even when an MHT of single Kalman filters based on the benign model was shown to be unable to track during maneuvers. Thus, the fact that the elemental filter probabilities regularly flowed to the benign elemental filter indicates that the multiple model filters were offering tracking capabilities that would be impossible with an MHT based only on benign-model Kalman filters. Furthermore, tracking performance was also superior to that of an MHT based only on aggressive-model Kalman filters, the best single-mode-filter MHT that was capable of tracking the target. The performance improvement was quantified by temporally averaging the rms errors over the entire simulation period and over only the benign portions of the flight, since the benefit of a multiple model structure is most apparent 
during the benign portions of flight. Analysis of the simulations shows that, during the periods of deferred decision-making, those times when the majority of the mixture probability does not agree with the actual target state, the multiple model structures had a tendency to exacerbate the error slightly, but the benefit of the multiple model structures vastly outweighs this performance penalty.

Extension to multiple targets $[16,23,26]$ and possibly using other distance metrics for the MRA [17] are receiving attention currently. Moreover, this research assumed the probability of detection $P_{d}$ to be unity, and performance under more realistic nonunity values warrants investigation as well.

\section{REFERENCES}

[1] Alspach, D.

A Gaussian sum approach to the multitarget

identification-tracking problem.

Automatica, 11, 3 (1975), 285-296.

[2] Bar-Shalom, Y., and Fortmann, T. E.

Tracking and Data Association.

Orlando, FL: Academic Press, 1988.

[3] Bar-Shalom, Y., and Li, X.

Multitarget-Multisensor Tracking: Principles and

Techniques.

Storrs, CT: YBS Publishing, 1995.

[4] Blackman, S. S.

Multiple-Target Tracking with Radar Applications.

Norwood, MA: Artech House, 1986.

[5] Blackman, S. S., and Popoli, R.

Design and Analysis of Modern Tracking Systems.

Norwood, MA: Artech House, 1999.

[6] Blackman, S. S., Dempster, R., and Nichols, T.

Application of multiple hypothesis tracking to multiradar air defense systems.

Multisensor Multitarget Data Fusion, Tracking and

Identification Techniques for Guidance and Control

Applications, NATO AGARD AG-337:96-120 (Oct. 1996).

[7] Blom, H. A. P., and Bar-Shalom, Y.

The interacting multiple model algorithm for systems with Markovian switching coefficients.

IEEE Transactions on Automatic Control, 33, 8 (Aug. 1988), 780-783.

[8] Kozak, M. C.

Multiple Model Methods for Cost Function Based Multiple Hypothesis Trackers.

MSEE thesis, Air Force Institute of Technology,

Wright-Patterson AFB, OH AFIT/GE/ENG/06-29, Mar. 2006.

[9] Kozak, M. C., and Maybeck, P. S.

Enhanced multiple model tracker based on Gaussian mixture reduction for a maneuvering target in clutter. In Proceedings of the SPIE, SPIE Annual International Defense and Security Symposium, Orlando, FL, Apr. 2006, paper 6236-16.

[10] Magill, D. T.

Optimal adaptive estimation of sampled stochastic processes.

IEEE Transactions on Automatic Control, 10, 5 (1965), 434-439.

[11] Maybeck, P. S.

Stochastic Models, Estimation and Control, vol 1.

Navtech, Arlington, VA, 1994.
[12] Maybeck, P. S.

Stochastic Models, Estimation and Control, vol 2.

Navtech, Arlington, VA, 1994.

[13] Maybeck, P. S., and Smith, B. D.

Multiple model tracker based on Gaussian mixture reduction for maneuvering targets in clutter.

In Proceedings of the 8th International Conference on Information Fusion, Philadelphia, PA, July 2005.

[14] Mazor, E., Averbuch, A., Bar-Shalom, Y., and Dayan, J. Interacting multiple model methods in target tracking: A survey.

IEEE Transactions on Aerospace and Electronic Systems, 34, 1 (Jan. 1998), 103-123.

[15] Olson, C. L.

FlightGear Flight Simulator.

http://www.flightgear.org.

[16] Pao, L. Y.

Multisensor multitarget mixture reduction algorithms for tracking.

AIAA Journal of Guidance, Control, and Dynamics, 17, 6 (Nov.-Dec. 1994), 1205-1211.

[17] Petrucci, D. J.

Gaussian Mixture Reduction for Bayesian Target Tracking in Clutter.

MSEE thesis, Air Force Institute of Technology, Wright-Patterson AFB, OH, AFIT/GE/ENG/06-01, Dec. 2005.

[18] Salmond, D. J.

Mixture Reduction Algorithms for Uncertain Tracking.

Royal Aerospace Establishment, Farnborough, UK, Technical Report 88004, Jan. 1988, DTIC Number ADA197641.

[19] Salmond, D. J.

Mixture reduction algorithms for target tracking. IEE Colloquium on State Estimation in Aerospace and Tracking Applications. London, UK: IEE Publishing, Dec. 1989.

[20] Salmond, D. J.

Tracking in Uncertain Environments.

Royal Aerospace Establishment, Farnborough, UK, Technical Memorandum AW 121, Sept. 1989, DTIC Number ADA215866. Taken from a D. Phil. dissertation of the University of Sussex.

[21] Salmond, D. J.

Mixture reduction algorithms for target tracking in clutter. SPIE Signal and Data Processing of Small Targets, 1305 (Apr. 1990), 434-445.

[22] Scott, D. W., and Szewczyk, W. F.

From kernels to mixtures.

Technometrics, 43, 3 (2001), 323-335.

[23] Singer, R., Sea, R., and Housewright, K.

Derivation and evaluation of improved tracking filters for use in a dense multi-target environment.

IEEE Transactions on Information Theory, IT-20 (July 1974), 423-432.

[24] Smith, B. D.

Multiple Model Adaptive Estimator Target Tracker for

Maneuvering Targets in Clutter.

MSEE thesis, Air Force Institute of Technology,

Wright-Patterson AFB, OH, AFIT/GE/ENG/05-18, Mar. 2005.

[25] Torelli, R., Graziano, A., and Farina, A.

IM3HT algorithm: A joint formulation of IMM and MHT for multi-target tracking.

European Journal of Control, 5, 1 (1999), 46-53. 
Vasquez, J., and Williams, J. L.

Improved hypothesis selection for multiple hypothesis tracking.

SPIE Signal and Data Processing of Small Targets, 5428, (Apr. 2004), 211-223.

[27] Williams, J. L.

Gaussian Mixture Reduction for Tracking Multiple Maneuvering Targets in Clutter.

MSEE thesis, Air Force Institute of Technology, Wright-Patterson AFB, OH, 2003.
[28]

Williams, J. L., and Maybeck, P. S.

Cost-function-based hypothesis control techniques for multiple hypothesis tracking.

In Proceedings of the SPIE, vol. 5428, 167-179, Orlando, FL, Apr. 2004, SPIE Annual International Defense and Security Symposium. Also Mathematical and Computer Modelling (Special Issue on Optimization and Control for Military Applications), 43 (2006), 976-989.

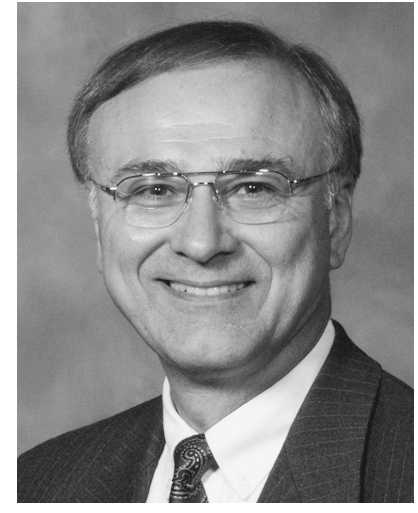

Peter S. Maybeck was born in New York, NY on February 9, 1947. He received the B.S. and Ph.D. degrees in aeronautical and astronautical engineering from M.I.T. in 1968 and 1972, respectively.

In 1968, he was employed by the Apollo Digital Autopilot Group of The C. S. Draper Laboratory, Cambridge Massachusetts. From 1972 to 1973, he served as a military control engineer for the Air Force Flight Dynamics Laboratory and then joined the faculty of the Air Force Institute of Technology in June of 1973. He is currently Professor of Electrical Engineering, responsible for the graduate sequence in estimation and stochastic control and for individual advanced digital filtering and control courses. He is author of over 140 papers on applied optimal filtering as well as the book, Stochastic Models, Estimation and Control (Academic Press, Vol. 1-1979, Vols. 2 and 3-1982; republished by Navtech in 1994 and 2001). Current research interests concentrate on using optimal estimation techniques for guidance and navigation systems, tracking, adaptive systems and failure detection purposes.

Dr. Maybeck is a member of Tau Beta Pi, Sigma Gamma Tau, Eta Kappa $\mathrm{Nu}$, and Sigma Xi. He was recipient of the DeFlorez Award (ingenuity and competence of research), the James Means Prize (excellence in systems engineering) and the Hertz Foundation Fellowship at M.I.T. in 1968. From 1975 to the current time, he consistently received commendations as outstanding Professor of Electrical Engineering at A.F.I.T. In December of 1978, he received an award from the Affiliate Societies Council of Dayton as one of the twelve outstanding scientists in the Dayton, Ohio area. In March of 1980, he was presented with the Eta Kappa Nu Association's C. Holmes MacDonald Award, designating him as the outstanding electrical engineering professor in the United States under the age of 35 (he had placed second in this national competition for 1977 as well). In 1985, he received the Frederick Emmons Terman Award, the highest national award to a Professor of Electrical Engineering given by the American Society of Engineering Education. In March 2000, he received the M. Barry Carlton Award, the annual award for the most outstanding technical article published in the IEEE Transactions on Aerospace and Electronic Systems. That same year, he also received the IEEE Third Millennium Medal for long-term service to the profession and to IEEE. He is a member of the AIAA, and he served for 25 years as the IEEE Dayton Section Student Activities Chairman and a member of the IEEE Executive Committee of Dayton, and he also served as Chairman of the local IEEE Automatic Control Group. 

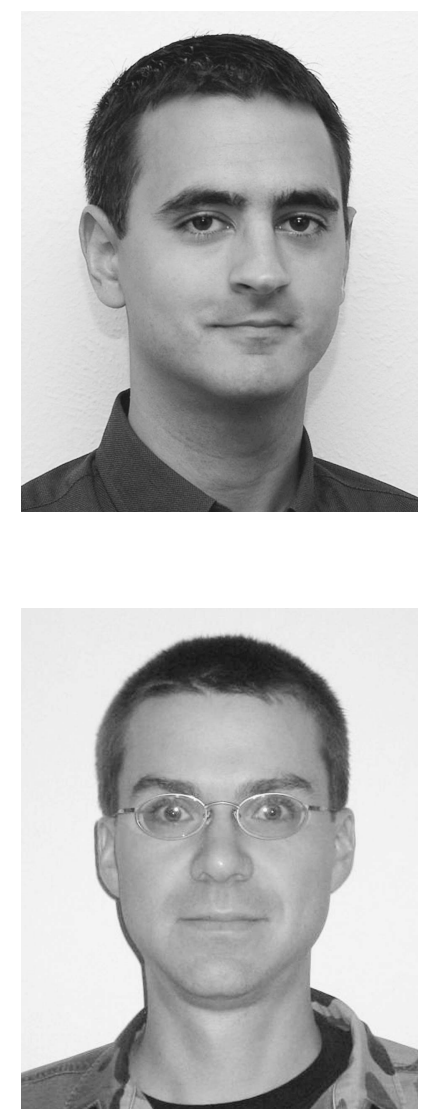

Matthew C. Kozak received the B.S. degree from the Georgia Institute of Technology, Atlanta, in 2001, and the M.S. degree from the Air Force Institute of Technology, Dayton, Ohio, in 2006, each in electrical engineering. He is currently working for the Modeling, Simulation, and Analysis branch of the Space and Missile Systems Center in Los Angeles, California.

Brian D. Smith received his M.S. in electrical engineering from AFIT, and his B.S. in electrical engineering from Purdue University.

He currently serves on active duty in the United States Air Force as an analyst and staff officer at the Air Force Operational Test and Evaluation Center. He received the 2006 U.S. Air Force Modeling and Simulation Award, Test \& Evaluation category, for his work on simulations supporting operational test of several Air Force system acquisition programs. Previously, he was a graduate student at the Air Force Institute of Technology (AFIT) specializing in guidance, navigation, and control systems. 